\title{
Efecto del desempeño ambiental sobre el riesgo de mercado en países emergentes: Caso de Brasil, Chile y México (Environmental performance and market risk in emerging countries: Brazil, Chile y México)
}

\section{'Alicia Fernanda Galindo Manrique}

Abstract. The production of wealth through industrial development and resource scarcity has let the adoption of environmental prevention practices by companies. The link between environmental performance and market response improving the strategic resource allocation and lowering operational risks, has become in one of the most relevant issues for academics and shareholders. The aim of this paper is to present the theoretical framework of the environmental performance and market risk in emerging countries, with the purpose to answer the research question: Which is the theoretical framework that support the link between these factors? Particularly, we focused this research in Brazil, Chile and Mexico, counties in economic expansion. At the end of this work we expose the conclusions presenting the principals business trends that are affected by this relationship.

Key words: emerging countries, environmental performance, market risk, resource allocation.

JEL: G10, Q51, Q56.

Resumen. La producción de riqueza a través del desarrollo industrial y la limitación de los recursos ha inducido el deterioro ambiental durante las últimas décadas, y esto a su vez, ha provocado que las organizaciones adopten prácticas de prevención medioambientales. El vínculo entre el desempeño ambiental y la respuesta del mercado ha atraído la atención de investigadores y del público inversionista permitiendo la disminución del riesgo, eficiencia operativa por la asignación estratégica de los recursos y la identificación de oportunidades de inversión que a largo plazo se traduce en rendimientos mayores. El objetivo de este trabajo es exponer los principales argumentos teóricos sobre el desempeño ambiental y riesgo en países emergentes. Particularmente se analizará el caso de Brasil, Chile y México. Al final del estudio se desarrollará la discusión del marco teórico en donde se expondrán las principales tendencias de negocios que afectan a la relación bajo estudio.

Palabras clave: desempeño ambiental, países emergentes, recursos estratégicos, riesgo de mercado. 


\section{Introducción}

En años recientes la atención hacia el medio ambiente ha cobrado mayor relevancia en los mercados internacionales (Aras, Aybars, \& Kutlu, 2010). El objetivo de las empresas se ha ampliado ya que ahora, no solo deben de generar riqueza y valor para sus accionistas, sino también deben de mostrar su responsabilidad hacia el medio ambiente.

La producción de riqueza a través del desarrollo industrial y la limitación de los recursos ha influido directamente en el deterioro ambiental durante las últimas décadas, y esto ha provocado que las organizaciones adopten prácticas de prevención medioambientales (Henri \& Journeault, 2010). Las prácticas en beneficio del medio ambiente forman parte de los pilares que constituyen la responsabilidad social en donde los negocios deben de asumir más compromiso con la sociedad y contribuir a una mejor calidad de vida (Carrol, 1999).

La premisa de la existencia del vínculo entre el desempeño ambiental y el desempeño económico en las corporaciones ha sido cuestionada y ha atraído la atención tanto de académicos, líderes de negocios y de accionistas. Esta atracción se debe a los resultados contundentes que se han presentado en la potencialización de utilidades y rendimientos económicos, por lo que la demanda de la información de desempeño ambiental por parte de los inversionistas ha tomado un rol crucial en los mercados financieros (Henri \& Journeault, 2010).

Los factores que han detonado el uso de la información del desempeño ambiental y han sensibilizado al mercado en cuanto al buen comportamiento sustentable, corresponden a la disminución de riesgos, la eficiencia operativa por la asignación estratégica de recursos y la identificación de oportunidades de inversión, que pueden tener una influencia positiva en el desempeño financiero de las empresas a largo plazo (Russo, 2016). La combinación de rendimiento y sustentabilidad en los países emergentes ha sido ejemplo de la sensibilización del mercado en las últimas décadas.

Los mercados emergentes son atractivos para los inversionistas, aunque representan una pequeña proporción de la inversión global directa(WEF, 2016). Existen dos factores clave según el Fondo Monetario Internacional (FMI) que consolidan la selección de mercados emergentes en portafolios de inversión. El primero se refiere al consenso entre la comunidad inversionista sobre el incremento en el consumo privado. El segundo factor hace referencia a la estabilidad económica y la confianza de los inversionistas sobre su crecimiento en el tiempo. 
Por su parte los mercados emergentes latinoamericanos no se han quedado atrás, y ejemplo de ello son los índices en mercados como Brasil con el ISE-Bovespa en el 2005, México con el Índice IPC Sustentable, creado en 2011 y en Chile el DJSI Chile Index en el 2015.El objetivo de éstos índices es dar a los inversionistas, mayor información sobre prácticas ambientales para integrar en sus portafolios empresas comprometidas con el medio ambiente y que reflejen rendimientos que compensen la inversión (Zhang, Zhou, \& Zhou, 2007).

Los países latinoamericanos han experimentado resiliencia, a pesar de la caída de las exportaciones y las turbulencias por la dependencia extranjera. La necesidad de potencializar la competitividad a través de la inversión y reformas estructurales ha sido fundamental para consolidar ganancias sociales y económicas. Chile es el país más competitivo en esta región con un ambiente macroeconómico sólido, un déficit pequeño, baja deuda pública y buen desempeño en sus mercados financieros, seguido por México (Schwab et al., 2014).

México se ha caracterizado por tener un ambiente macroeconómico estable y un mercado interno fuerte que permite el desarrollo de economías de escala. Por otro lado, Brasil ha experimentado un declive en su crecimiento debido a factores como a infraestructura, ineficiencia de las instituciones y corrupción en el gobierno. Sin embargo, este país se sigue beneficiando de sus fortalezas, especialmente del tamaño de su mercado, comunidad de negocios sofisticada, innovación y actividades de investigación que agregan valor (Schwab et al., 2014).

Bajo este contexto, el objetivo del presente trabajo es exponer las principales posturas y argumentos teóricos acerca del desempeño ambiental y riesgo de mercado; así como las tendencias y avances sobre este vínculo. Para cumplir con dicho objetivo se llevará a cabo una revisión documental y un análisis de las principales teorías en las que yacen los efectos de esta relación, buscando contestar a la pregunta: ¿Cuál es el marco teórico que establece el efecto del desempeño ambiental sobre el riesgo de mercado en países emergentes? Se estudiarán los avances que existen de Chile, Brasil y México, países emergentes con crecimientos potenciales en sus mercados de capitales. Cabe destacar que existe un área de oportunidad en los estudios realizados en países latinoamericanos con la característica de estar en vías de desarrollo. Así mismo, la aportación de este proyecto de investigación contribuirá a la construcción de la relación entre el desempeño ambiental y financiero.

El presente trabajo de investigación tiene la siguiente estructura: en la sección 2 se presentan los antecedentes del desempeño ambiental y riesgo de 
mercado, en la sección 3 se establece el desarrollo del vínculo en los países emergentes bajo estudio, y por último en la sección 4 y 5 se presentan las discusiones y resultados del estudio retomando implicaciones y perspectivas del desempeño ambiental y riesgo de mercado.

\section{Antecedentes}

La relación entre el compromiso social de la empresa y su rentabilidad ha sido una preocupación científica desde 1970. Investigadores como Margolis, Elfenbein, \& Walsh, (2007) y Orlitzky, Schmidt, \& Rynes, (2003) han presentado amplia evidencia en la literatura existente, llegando a la conclusión de que el mercado premia las actividades sociales de las organizaciones. La Responsabilidad Social Empresarial (RSE) representa una estrategia administrativa eficiente (Vives, 2014), y puede ser un factor crucial en el éxito de la empresa.

La RSE es una inversión en el futuro de las empresas y es una práctica que debe de ser planeada, supervisada y evaluada regularmente. El uso correcto de esta estrategia puede potencializar la reputación de la organización (Falck \& Heblich, 2007). Desde el punto de vista de la oferta, la reputación de las empresas atrae, retiene y motiva a los empleados. Por otro lado, desde la perspectiva de la demanda incrementa el precio de la marca y por consiguiente el valor de la empresa.

El término de RSE fue acuñado por Bowen (1953), quien argumentó de manera normativa que: "Se refiere a la obligación de los hombres de negocio a perseguir aquellas políticas o decisiones que siguen líneas de acción deseables en términos de los objetivos y valores de la sociedad" (p.6). Una década después, diversos autores, incluyendo a Davis, (1975) McGuire, Sundgren, \& Schneeweis, (1988) y (Walton, 1967) retomaron el desarrollo de éste concepto haciendo referencia, no solo a los "hombres de negocio", sino también a diversos actores e interesados relacionados con la empresa. Esta idea fue completamente refutada por Friedman, (1970) quien rechazó de manera contundente el compromiso social.

Este discernimiento en la literatura contempla dos enfoques ampliamente estudiados. El enfoque de los "shareholders" o accionistas, encabezado por Friedman, (1970) y el enfoque de los "stakeholders" o partes interesadas y agentes dirigido por (Freeman, Wicks, \& Parmar, (1984). Desde el punto de vista de Friedman, los administradores en un sistema económico libre están obligados 
por contrato a maximizar el valor de la empresa para los accionistas, y si buscan cualquier mejora social, lo podrán hacer como individuos privados sin afectar el costo de la empresa Friedman, (1970). Por otro lado, Freeman propone la capitalización de las externalidades al contemplar en su modelo los diversos agentes que afectan a las organizaciones (Falck \& Heblich, 2007). Estos agentes están formados por proveedores, clientes, colaboradores, ambientalistas, gobierno y la sociedad (Freeman, Wicks, \& Parmar, 1984)

La propuesta realizada por Freeman (1984) se complementa con la "Teoría instrumental de los stakeholders" desarrollada por Thomas Jones (Berman, Wicks, Kotha, \& Jones, 1999) en donde sintetiza el concepto de los agentes, la teoría económica y la ética organizacional. Dentro del marco agente-principal desarrollado por Jensen \& Meckling, 1976), Jones establece que la empresa es una red de relaciones contractuales implícitas y explícitas entre los administradores y las partes relacionadas.

El autor sugiere que tratar justamente a los "stakeholders" (agentes) mostrándoles buena voluntad y fe establece una reputación positiva que conlleva una ventaja competitiva. Dicha ventaja competitiva se traduce en la reducción de costos y los beneficios ganados por mantener una relación de confianza mutua.

Retomando los fundamentos de la RSE, esta puede ser usada estratégicamente como vínculo conciliador para realizar planes que satisfagan tanto a los accionistas como a las partes relacionadas en el plano social y ambiental.

En el marco ambiental la generación de riqueza a través del uso limitado de los recursos ha atraído la atención de líderes académicos e investigadores. El desarrollo industrial ha provocado un efecto combinado entre prosperidad económica y degradación ecológica (Henri \& Journeault, 2010). En los últimos años las industrias y organizaciones han adoptado prácticas de prevención medioambientales (Al-Najjar \& Anfimiadou, 2012) propiciadas por una fuerte presión por parte de los inversionistas quienes fomentan la conciencia social como práctica en los negocios.

La relación económica ambiental ha sido estudiada desde 1970 y su correspondencia positiva no siempre ha recibido aceptación universal (Murphy, 2002). Tal es el caso de investigaciones realizadas que establecen que la inversión en actividades ecológicas representa un costo que debe de ser minimizado siempre que sea posible.

Estudios hacen referencia al punto de vista de los inversionistas quienes ven los gastos de control pro ambientales, como una pérdida de recursos que 
podrían ser invertidos para generar rentabilidad y que a su vez no premian el comportamiento responsable de las empresas (Mahapatra, 1984). Por otro lado, evidencia en la literatura ecológica-financiera, asocia la decisión de los inversionistas al tomar en cuenta las buenas prácticas ambientales con la percepción de disminución de riesgo de mercado, al seleccionar empresas que cumplan con este perfil (Mahapatra, 1984).

Esta percepción positiva ocasiona que los mercados financieros estén dispuestos a aceptar menores primas de riesgo sobre capital o a permitir que la empresa adquiera altos niveles de endeudamiento. En ambos casos, el reflejo económico se ve plasmado en la disminución del costo de capital y el incremento de las utilidades de la organización (Murphy, 2002).

Sobre esta línea de investigación algunos autores establecen que la empresa al realizar inversiones estratégicas que tengan un impacto favorable en el medio ambiente, promueven la mitigación de riesgos futuros evitando impactos financieros inciertos (King \& Shaver, 2009).

La reducción de daños potenciales libera recursos económicos que pueden ser utilizados estratégicamente en la empresa, otorgando dividendos para los accionistas, promoviendo la inversión interna y generando posibles adquisiciones. El mercado recompensa cada una de estas actividades incrementando el precio de la acción y el número de accionistas en el mercado.

Las empresas están adquiriendo mayor responsabilidad corporativa y están comprometiéndose a la conservación del medio ambiente por diferentes medios como la auto-regulación, la creación de políticas ambientales corporativas, códigos de práctica voluntarios, y mediante auditorías y reportes públicos. La práctica de estas iniciativas se debe a la incorporación de sistemas de gestión ambiental.

Estos sistemas son relativamente nuevos y propician una serie de lineamientos para la conservación del medio ambiente, uso correcto de las materias primas, residuos generados y penalizaciones por incumplimiento a la norma (British Standards Institution, 2016).

El surgimiento de los estándares de gestión ambiental data de la década de los 90. La primera norma británica de gestión ambiental denominada BS7750 fue pionera en el establecimiento de protocolos para el control de emisiones en la industria europea (La norma británica BS7750 sido reemplazada por la norma internacional ISO 14000). Al mismo tiempo la Unión Europea desarrolló el Plan de Gestión y Auditoría Ecológica EMAS para proporcionar a lar organizaciones 
otra herramienta para demostrar a los consumidores su compromiso con el medio ambiente (Iraldo, Testa, \& Frey, 2009).

El compromiso de las empresas con la sustentabilidad se ve reflejado al reportar operaciones y su impacto con el medio ambiente en informes anuales que complementan la información de desempeño económico de la empresa. Esta sensibilización está generando un cambio en la cultura corporativa obteniendo un interés real de los gerentes, accionistas, investigadores y clientes de la entidad. (Holdgate, 1996). Estudios recientes subrayan la importancia del cuidado ambiental y la convicción de los consumidores de pagar más por productos eco-eficientes (Rosewicz,1990).

Los accionistas reflejan una opinión similar, recomendando la inversión en gastos de capital que contribuyan con la preservación de la naturaleza, en segundo lugar, la innovación y creación de productos verdes, y en tercer lugar el pago de dividendos altos (Epstein \& Geiger, 1994) Éstas tendencias convergen en la necesidad de generar estudios que refuercen el vínculo entre el desempeño ambiental y financiero de las entidades. Los países desarrollados han capitalizado estos esfuerzos en las prácticas de los negocios. Sin embargo, las economías emergentes a pesar de sus crecimientos, no han consolidado la cultura y desempeño medio ambiental.

\section{Metodología}

El objetivo de este trabajo es exponer las principales posturas teóricas acerca de la relación y efectos del desempeño ambiental y riesgo de mercado en países emergentes. Para cumplir con este objetivo se llevó a cabo una revisión de literatura y estado del arte sobre las teorías que establecen el marco base para contestar la siguiente pregunta de investigación: ¿cuál es el marco teórico que establece el efecto del desempeño ambiental sobre el riesgo de mercado en países emergentes?

El presente trabajo es de tipo cualitativo y descriptivo, ya que se basa en la revisión documental sobre las teorías principales relacionadas con el desempeño ambiental, rendimiento financiero y riesgo de mercado. Se desarrolló la teoría fundamentada como metodología de investigación cualitativa cuyo objetivo es la descripción sistemática de los estudios para generar conceptos como resultado del método (Hernández et al., 2010). 


\section{Marco teórico}

Investigaciones previas sobre la relación del desempeño económico y ambiental han arrojado resultados mixtos a lo largo de la historia. Bragdon y Marlin (1972) argumentaron que el abatimiento de la contaminación y la rentabilidad son compatibles. Los autores encontraron relación positiva entre medidas económicas (utilidad por acción y retorno sobre capital) y los rankings sobre comportamiento ambiental, desarrollados por el Council de Prioridades Económicas (CPE) para empresas de la industria del papel y pulpa.

Siguiendo con la industria del papel, Spicer (1978) utilizó empresas de este nicho para medir la asociación entre cinco variables de crecimiento financiero. Entre ellas destacan la rentabilidad, tamaño de la empresa, riesgo total, riesgo sistemático y la razón precio/utilidad.

Estas variables las comparó con el índice de contaminación de la CPE y sus resultados concluyeron que solamente el riesgo sistemático, y la razón precio-utilidad fueron estadísticamente significativas.

Aunque los dos estudios anteriormente mencionados fueron criticados por los pequeños tamaños de muestras que se utilizaron y errores de medición, los hallazgos encontrados fueron consistentes con la idea de que el buen desempeño ambiental y económico se complementan. Aunado a este concepto, dichas investigaciones relacionaron la idea sobre las externalidades, las cuales reducían el riesgo al cual los mercados de capitales están altamente sensibles (Narver, 2004).

Adicionalmente, si la contaminación representa recursos que son deficientes o mal usados por la empresa, la eliminación de dicho desperdicio e ineficiencias benefician tanto al medio ambiente como a las utilidades. (Porter y Van der Linde, 1995a, 1995b)

Por otro lado, investigaciones realizadas encontraron que la asociación definida anteriormente es estadísticamente insignificante. Rockness et al, (1986) examinó los residuos tóxicos de la industria química utilizando datos ambientales y financieros provistos por el Congreso de Estados Unidos en 1979. Probando la asociación de dos variables relacionadas a los residuos tóxicos y doce indicadores financieros, que representaban el desempeño económico de la empresa, Rockness no encontró una relación estadísticamente significativa.

Freedman y Jaggi (1992) examinaron la relación a largo plazo entre el desempeño ambiental y económico utilizando el cambio porcentual de tres 
medidas de contaminación y razones financieras como proxis empíricos de desempeño. Los resultados no apoyaron esta hipótesis descartando la asociación significativa.

Desde otro punto de vista, el desempeño ambiental va de la mano con la divulgación de la misma para dar credibilidad a las organizaciones. Sin embargo, diferentes autores han demostrado que no existe asociación significativa entre estas dos variables. Ingram y Frazier (1980) realizaron un estudio comparativo de los índices de divulgación ambiental de los reportes anuales versus el ranking realizado por la CPE. No se identificó asociación. Freedman y Jaggi (1992) también reportaron asociación insignificante utilizando reportes anuales de formatos 10-K (Formato de reporte requerido por la SEC (Securities Exchange Commission), que otorga un resumen del desempeño financiero de la empresa. Se reporta de forma anual).

En conjunto con estas investigaciones, Rockness (1986) llevó a cabo un experimento de campo en el cual participaron analistas financieros, miembros destacados de organizaciones pro ambientales, autoridades de protección al ambiente y estudiantes. En esta prueba, el grupo de sujetos evaluó la información ambiental contenida en los reportes anuales de las empresas, y como resultado se obtuvieron coeficientes de correlación negativos, implicando que los sujetos evaluaron los peores desempeños ambientales como algo positivo y viceversa. Como resultado, Rockness refirió que el grupo del experimento fue mal guiado por la información presentada.

Li (2010) decretó en su estudio que, si se asume que el buen desempeño ambiental reduce la exposición de la empresa a costos futuros, entonces la presentación de la información debe de ser percibida de forma positiva para los inversionistas. Por lo tanto, empresas con buen desempeño ambiental deben de divulgar más en calidad y cantidad, que empresas con desempeños pobres. Por otro lado, si la difusión de información es encaminada a ser utilizada en litigios en contra de la empresa, el buen desempeño ambiental tenderá a ser minimizado.

Estudios realizados, sobre la relación económica y ambiental, han utilizados medidas de mercado para determinar si el riesgo sobre el precio de la acción se ve afectado. Uno de estos estudios, fue el realizado por Shane y Spicer (1983) en donde utilizaron la Teoría de Eventos. Los resultados documentaron una reacción negativa del mercado durante los dos días posteriores a la emisión de los reportes ambientales de la CPE. Del mismo modo, Wartick y Cochran (1985) concluyeron que un portafolio de inversión formado por empresas que 
divulgan información ambiental en sus reportes, en donde establecían costos futuros por prevención de contaminación, experimentaron rendimientos mensuales bajos que empresas cuyos costos esperados fueron bajos.

Richardson et al. (2012) observó que la divulgación social-ambiental se comportaba diferente que la información financiera general, asociada al costo de capital. Esta investigación reportó una relación negativa significativa entre el nivel de información financiera detallada en reportes y el costo de capital. Si se asume una relación inversa entre el costo de capital y el precio de la acción, como lo sugiere el modelo de dividendos descontados, los resultados de Richardson (2012) infieren que el incremento en la divulgación de información ambiental está asociado con precios de acción bajos.

Sin embargo, esta evidencia no es consistente con la noción de que la divulgación discrecional reduce los costos de la información asimétrica o que la presentación de información dispare un efecto de preferencia favorable por parte de los inversionistas. Este efecto se le conoce también como Green pricing en donde los inversionistas están dispuestos a pagar más por empresas responsables con el medio ambiente (Tietenberg, 1998).

Por otra parte, investigaciones realizadas en países emergentes han expuesto la constante regulación de los gobiernos con el objetivo de castigar conductas que afecten al medio ambiente. Existe también una presión por parte de los consumidores, ya que de acuerdo a Nieto y Fernández (2004) cada vez más existe una sensibilización sobre la tendencia de consumo de productos provenientes de empresas responsables.

Charlo et al. (2003) decreta que existe una conciencia verde de los inversionistas en temas ambientales y que ésta forma parte de los criterios de inversión a largo plazo en los mercados de capitales. Esta conciencia verde y su impacto en los mercados constituye el fundamento principal presentado en este trabajo particularmente para los países de México, Chile y Brasil.

Como parte del presenta trabajo de investigación documental, a continuación, se presenta en la siguiente tabla la revisión literaria realizada que engloba los principales estudios de desempeño ambiental y desempeño financieros, así como el estudio de la relación existente entre el riesgo de mercado y la volatilidad en los precios de las acciones de empresas comprometidas con el desempeño ambiental. 
Tabla 1. Estudios Empíricos sobre el desempeño ambiental y perfil de riesgo

\begin{tabular}{|c|c|}
\hline Autor & Estudio \\
\hline $\begin{array}{l}\text { Christmann } \\
\quad(2000)\end{array}$ & $\begin{array}{l}\text { Empresas químicas con capacidad de innovación en la reducción } \\
\text { de contaminación a través de tecnología reconocieron ahorros en } \\
\text { costos significantes. Reducción del perfil de riesgo. }\end{array}$ \\
\hline $\begin{array}{l}\text { Cohen et al. } \\
\text { (1995) }\end{array}$ & $\begin{array}{l}\text { Portafolios de industrias balanceadas tipificados como "contami- } \\
\text { nadores bajos" en el S\&P obtuvieron mayores rendimientos en sus } \\
\text { acciones que portafolios con "contaminadores altos". }\end{array}$ \\
\hline $\begin{array}{l}\text { Dowell et al. } \\
\text { (2000) }\end{array}$ & $\begin{array}{l}\text { Empresas con estándares internacionales sobre el medio } \\
\text { ambiente más estrictos que las regulaciones locales tuvieron ma- } \\
\text { yores rendimientos de mercado que empresas con estándares, } \\
\text { iguales o menores sobre lo requerido. }\end{array}$ \\
\hline $\begin{array}{l}\text { Gottsman } \\
\text { y Kessñer (1998) }\end{array}$ & $\begin{array}{l}\text { Portafolios de empresas "buenas con el medio ambiente" obtuvieron } \\
\text { rendimientos mayores en el S\&P, que portafolios "contaminantes". }\end{array}$ \\
\hline $\begin{array}{c}\text { Hart y Ahuja } \\
\quad(1995)\end{array}$ & $\begin{array}{l}\text { La prevención de contaminación y las iniciativas de reducción de } \\
\text { emisiones mejoran indicadores como rendimiento de capital, acti- } \\
\text { vos y ventas ROE, ROA y ROS. }\end{array}$ \\
\hline $\begin{array}{l}\text { Klassen } \\
\text { y McLaughlin } \\
\text { (1996) }\end{array}$ & $\begin{array}{l}\text { Empresas que recibieron premios por su desempeño ambiental in- } \\
\text { crementaron su valor de mercado mientras que la mala publicidad } \\
\text { tuvo el efecto contrario. }\end{array}$ \\
\hline $\begin{array}{l}\text { Konar y Cohen } \\
\text { (2001) }\end{array}$ & $\begin{array}{l}\text { El desempeño ambiental correlacionado con el valor de activos } \\
\text { intangibles y la reducción de desechos tóxicos químicos resultaron } \\
\text { en un incremento del valor de mercado de la empresa. }\end{array}$ \\
\hline Nehrt (1996) & $\begin{array}{l}\text { Empresas manufactureras de papel que realizaron inversiones en } \\
\text { tecnología para reducir la contaminación experimentaron tasas } \\
\text { anormales de crecimiento. }\end{array}$ \\
\hline $\begin{array}{l}\text { Russo y Fouts } \\
\text { (1997) }\end{array}$ & $\begin{array}{l}\text { El rendimiento sobre activos (ROA) de las empresas mejora mien- } \\
\text { tras mejora su desempeño ambiental. }\end{array}$ \\
\hline Stanwick (1998) & $\begin{array}{l}\text { Empresas con excelente reputación social responsable y con ba- } \\
\text { jas emisiones experimentaron utilidades altas que empresas con } \\
\text { altas emisiones. }\end{array}$ \\
\hline White I (1995) & $\begin{array}{l}\text { Portafolios de empresas con reputación ambiental buena ganaron } \\
\text { rendimientos altos que portafolios con empresas de reputación } \\
\text { neutral o mala. }\end{array}$ \\
\hline
\end{tabular}

Fuente: Elaboración propia con datos de Murphy (2012) 


\section{Mercados emergentes y desempeño ambiental}

Los mercados emergentes han mostrado un crecimiento sostenido en tamaño, influencia, integración económica, así como un incremento en la conciencia ambiental. De acuerdo al Fondo Monetario Internacional FMI se espera que en el 2020 las economías emergentes representen el 85 \% de la población global por lo que la asignación de recursos en estos mercados representa una opción atractiva para obtener rendimientos altos. Países que combinan oportunidades de inversión con políticas ambientales e índices sustentables de mercado son: México, Brasil y Chile.

En la Figura 1 se puede observar como los países emergentes han duplicado su crecimiento en relación a las economías avanzadas. De acuerdo al Fondo Monetario Internacional, el crecimiento tendrá una tendencia alcista para los próximos diez años, lo cual representa un riesgo en función a la escases de recursos y al mismo tiempo una oportunidad para el desempeño ambiental.

Figura 1. Crecimiento de mercados emergentes

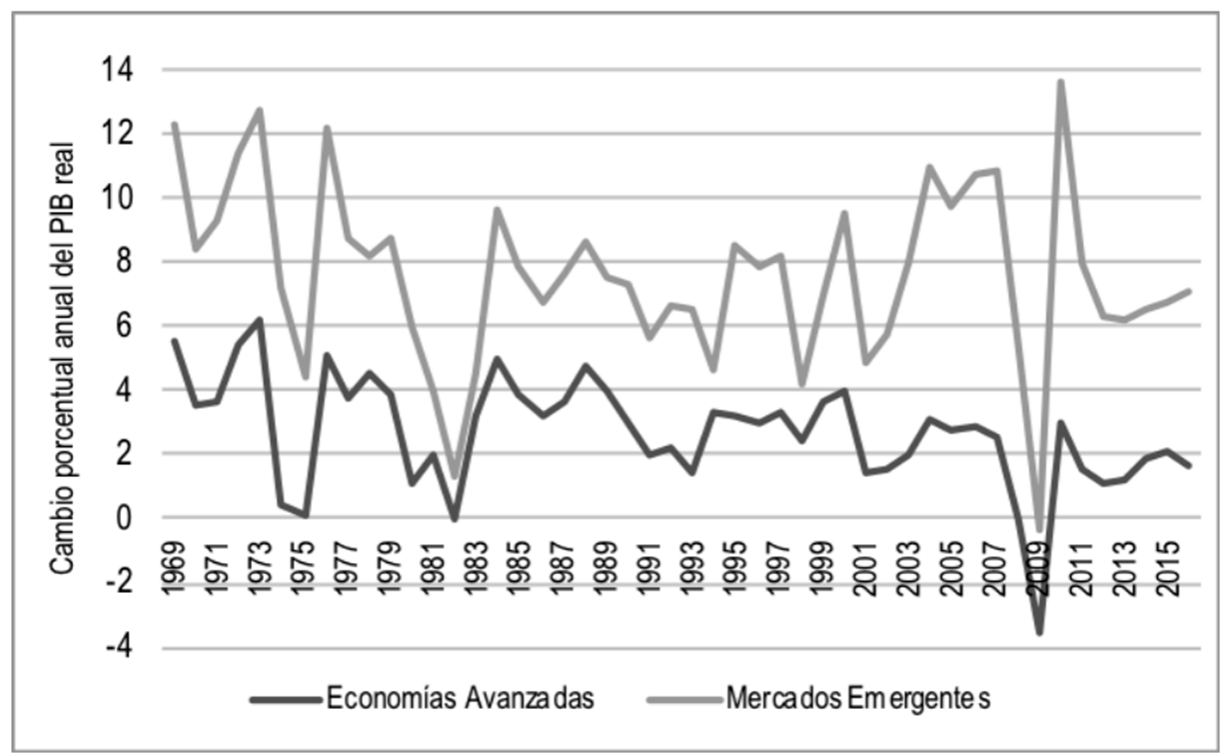

Fuente: Elaboración propia con datos del FMI (2016).

México se encuentra entre las mayores economías de la Organización para la Cooperación y el Desarrollo Económico OCDE. El país experimentó un 
crecimiento promedio durante la última década y se recuperó rápidamente de la crisis mundial, impulsado principalmente por las exportaciones hacia Estados Unidos. (OCDE, 2016). Durante este periodo, México fortaleció su política ambiental y registró avances en la conservación de la biodiversidad y los recursos naturales, así como en la gestión de los recursos hídricos. También puso de manifiesto el liderazgo en la cooperación internacional en cuanto al cambio climático. Sin embargo, la rápida urbanización, el crecimiento demográfico y el aumento en el consumo, como resultado de mayores ingresos, está generando una serie de retos ambientales. (OCDE, 2016).

El Índice de Competividad Global (ICG, 2016) sitúa a México en el lugar número 51 de 138 con un Producto Interno Bruto (PIB) de $\$ 1,144.3$ billones de dólares representando el $1.6 \%$ del PIB mundial. El país mejoró seis posiciones con respecto del año anterior debido a las ganancias en la eficiencia de mercado. La competencia doméstica y extranjera en el mercado de bienes mejoró significativamente como reflejo del resultado de reformas de comercio. El mercado laboral demostró flexibilidad e incentivos y los mercados financieros lograron reflejar éstos beneficios. (ICG, 2016). Aunque la economía mexicana ha sido golpeada por la caída de los precios del petróleo, comercio global débil y baja producción industrial, México sigue siendo una de las economías más competitivas en la región.

Por otro lado, Brasil ocupa el lugar 81 de 138 de acuerdo al reporte del índice de competitividad y tiene un PIB de \$1,772.6 billones de dólares representando el $2.81 \%$ a nivel mundial (ICG, 2016). El país se encuentra en depresión. La tasa de crecimiento se desaceleró desde el 2006 al 2016 al pasar de $4.5 \%$ a $2.1 \%$. La crisis económica como resultado de la caída del precio de los subyacentes y la falta de políticas, han ocasionado que el progreso económico esté en pausa (The World Bank, 2016).

El panorama a mediano plazo de Brasil dependerá de los ajustes en reformas y promulgación de leyes que propicien el crecimiento. El reto primordial del país es elevar la productividad y competitividad para lograr crecimiento a largo plazo. Con la recesión del crecimiento de factores clave como petróleo, mercado laboral y los subyacentes, el crecimiento necesitará ser basado en inversiones altas en los mercados. El país enfrenta el reto de encontrar maneras de combinar los beneficios del crecimiento en la agricultura, protección ambiental y desarrollo sustentable (The World Bank, 2016). Brasil tuvo un rol clave en la Conferencia de las Partes COP 21 en París demostrando su liderazgo en negociaciones in- 
ternacionales de cambio climático a través de la reducción de gases de efecto invernadero entre $36.1 \%$ y $38.9 \%$ para el 2020 .

Con respecto a Chile, este país ha sido una de las economías latinoamericanas de más rápido crecimiento. Ocupa el lugar 33 de 138 en el índice de competitividad (IGC, 2016) y tiene un PIB de $\$ 240.2$ billones de dólares con $0.37 \%$ del PIB a nivel mundial. Sin embargo, después de la expansión económica de 2010 a 2012, el PIB decayó $1.9 \%$ en el 2014 y $2.1 \%$ en el 2015, como resultado del atraso en el sector minero debido al fin del ciclo de inversión, la caída del precio del cobre y el consumo privado. Las reformas estructurales de Chile lo han mantenido como referencia en Latinoamérica de modelos de buen gobierno y sustentabilidad (The World Bank, 2016).

La relación entre el desempeño ambiental y riesgo de mercado puede diferir dependiendo del régimen regulatorio de cada país, cultura, comportamiento de los clientes, el tipo de industria y tamaño de la empresa. Tomando en cuenta estas características, no se ha llevado a cabo un estudio comparativo entre empresas públicas de México, Chile y Brasil, que sólo mida el efecto ambiental y riesgo en estos países. Esto representa un área de oportunidad en el que futuras investigaciones podrán soportar su hipótesis a desarrollar.

\section{Discusión}

Realizando una síntesis de las investigaciones científicas trascendentes analizadas en este artículo se puede encuadrar el tema de eco-eficiencia y riesgo de mercado, como parte de la tendencia de las organizaciones al incluirla en sus objetivos estratégicos para generar valor.

El estudio de esta relación data desde 1970 en donde la comunidad científica empezó a estudiar la eficiencia en la asignación de recursos con el objetivo de evitar desperdicios, a su vez, estos ahorros se reflejaban en el incremento de utilidades de la empresa y, por lo tanto, eran bien vistos por los inversionistas, accionistas y partes relacionadas.

La creación de valor de las empresas es un proceso que requiere la vinculación de decisiones de distribución de recursos y la estrategia de crecimiento a largo plazo, teniendo como punta de lanza la responsabilidad con social, en especial con el medio ambiente.

La conciencia ambiental en los inversionistas ha potencializado la inversión socialmente responsable en los mercados. Así mismo, la relevancia de este 
tema ha originado posibles líneas de investigación como el screening investment (inversiones a través de filtros sociales), el papel de la divulgación de información a través del GRI (Global Reporting Initiative) y la convergencia de los países emergentes al potencializar los rendimientos del mercado adoptando prácticas medio ambientales.

Las bolsas de valores, impulsadas por estas iniciativas, también han estado apoyando los modelos de negocios verdes (Makower, 2017). Cerca del $60 \%$ de las bolsas de valores mundiales ha implementado, o están en el proceso de desarrollar requerimientos ambientales para empresas que quieran cotizar en los mercados.

Aunque hoy en día, cualesquiera de los requerimientos son voluntarios, cada uno de los formatos solicitados tienen una amplia cobertura y cubren la mayoría de las industrias en los Estados Unidos. En la Figura 2 se presenta el número de bolsas de valores con requerimientos medio ambientales y su evolución a través del tiempo.

De acuerdo al reporte "State of Green Business 2017" desarrollado por Trucost -Trucost es motor de información que provee datos y modelos sustentables de negocios, así como estrategias de inversión. Actualmente fue adquirida por S\&P Dow Jones Indices-, se muestra un alejamiento claro y creciente de modelos de negocios que actividades que dañan al medio ambiente y que no son sustentables (Makower, 2017).

Inversionistas que han dejado de invertir en combustibles fósiles ronda los $\$ 5$ trillones de dólares. Actualmente existen 688 instituciones y cerca de 58,399 individuos a lo largo de 76 países que se han comprometido a des invertir en estos combustibles motivados por preocupaciones éticas y financieras. Estas métricas demuestran que los inversionistas están fomentando cada vez más actividades sustentables en beneficio del medio ambiente y que los modelos de negocios de las empresas están respondiendo a esta llamada del mercado, ofreciendo productos y servicios verdes (se refiere que contribuyen y benefician al medio ambiente. En inglés el término es greener). 
Figura 2. Número de Bolsas de Valores con requerimientos medio ambientales

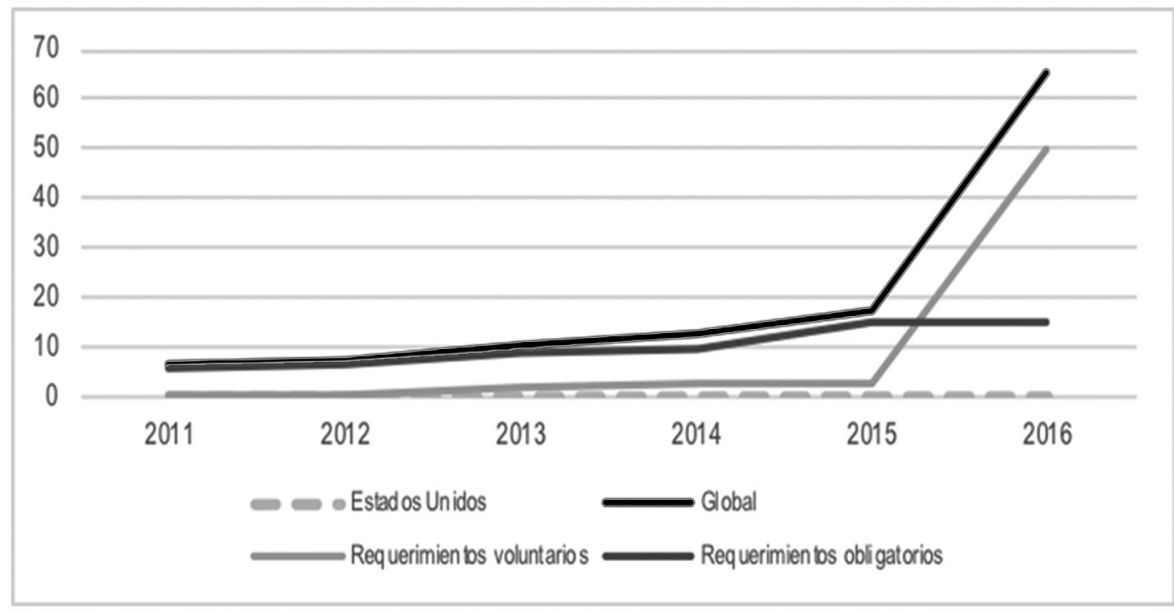

Fuente: Elaboración propia con datos de US FIS (2016).

Dentro del panorama global, los países emergentes han demostrado su interés en el medio ambiente al incorporar factores sustentables en sus mercados financieros. En los últimos diez años, en contraste con mercados desarrollados como Europa y Estados Unidos, las economías de los mercados emergentes han desarrollado oportunidades de inversión robustas atrayendo flujos de capital de países desarrollados. Existen dos factores que han incrementado esta participación.

El primero es el consenso creciente entre economistas expertos sobre el crecimiento potencial de dichos mercados emergentes. Mientras que las economías desarrolladas se encuentran en etapas de consolidación, numerosos países emergentes han demostrado finanzas públicas sanas y un incremento en el consumo privado (Marritt-Alers \& Giese, 2013).

El segundo factor es la confianza de los inversionistas y la habilidad de los mercados emergentes de crecer a través del tiempo (Morck, Yeung, \& Wayne, 2000). Las economías emergentes están enfrentando retos provenientes de la escasés de recursos y el crecimiento de la población en una mayor magnitud que los países desarrollados al inicio del siglo XX. Esto ha generado modelos de negocios que usan los inversionistas para identificar oportunidades, que en muchas ocasiones pueden quedar como estrategias incompletas, al no cubrir todo el rango de variables que son importantes al analizar mercados emergentes. 


\section{Mecanismos fiscales y autoridades que regulan el desempeño ambiental}

Otro elemento que entra en discusión es el marco regulatorio y los mecanismos fiscales en América Latina que cumplen con el objetico de regular el medio ambiente y su desempeño. Al entrar en materia fiscal, el impuesto a la gasolina representa entre el $3 \%$ y $3.5 \%$ del PIB según el país. De acuerdo al estudio del Comisión Económica para América Latina y el Caribe CEPAL, más del $90 \%$ de la recaudación de carácter ambiental proviene de impuestos verdes sobre vehículos y combustibles como gasolina y diesel (Quiroga Martinez, 2007).

En América Latina se observa una utilización más frecuente de instrumentos fiscales de carácter ambiental (Fanelli, Jiménez, \& Azúnaga, 2015). Brasil en el 2013, como parte de sus elementos ambientales en la reciente reforma tributaria, estableció el Impuesto sobre los productos Industrializados (IPI), así como la reducción de impuestos y tarifas a la energía eléctrica. El uso de los recursos se aplica a la consolidación fiscal.

La coordinación de políticas ambientales en Brasil es a través del Ministerio del Medio Ambiente en cooperación con el Sistema Nacional Ambiental. Brasil es pionero en la implementación de índices sustentables como se ha comentado en párrafos anteriores y también ha sido voluntario en la prueba piloto de medición de datos sustentables, entre ellos de medio ambiente. Este programa se encuentra ligado al Instituto de Geografía y Estadística (IBGE) que es la agencia que produce información estadística de la población y del desempeño ambiental del país.

Por su parte Chile en el 2014 estableció un Impuesto sobre el Carbono y también tiene efecto en la consolidación fiscal. Chile, en función de la reforma tributaria del 2014, propuso la creación de un grupo de impuestos verdes que tienen como objetivo tener un impacto regional y global sobre las emisiones contaminantes. Los tres impuestos son 1) impuesto a la importación de vehículos livianos diésel; 2) impuesto a la emisión de fuentes fijas de material particulado, óxido de nitrógeno y dióxido de azufre, y por último 3) impuesto a la emisión de fuentes fijas de dióxido de carbono que tengan potencia térmica igual o superior a 50 MWt (megavatios térmicos) (Katz, 2014).

Este último corresponde a un impuesto pionero en América Latina en donde se aplica una tasa impositiva de $\$ 5$ usd/ $/ \mathrm{CO}_{2}$ que se encuentra en el rango bajo de las legislaciones de otros países y muy por debajo de algunas estimaciones del costo social del carbón (Fanelli et al., 2015). 
En Chile la autoridad encargada de regular las imposiciones a tributos verdes es el Ministerio del Medio Ambiente y a través de la Ley 20.870 aprobada en septiembre de 2014, estableciéndose tres impuestos ambientales: a) primera venta de vehículos en donde se mide el rendimiento urbano a través del NOx (óxidos de nitrógeno), b) contaminantes globales a las emisiones de $\mathrm{CO}_{2}$ y c) contaminantes locales. Estos impuestos operan desde el 01 de enero de 2017. Esta ley tiene un plan de descontaminación ambiental cuyo objetivo es reducir las emisiones en un $40 \%$ de material particulado, $51 \%$ de óxido de nitrógeno y $61 \%$ de dióxido de azufre.

En México en el 2014 como parte de las reformas sexenales provenientes del Plan Nacional de Desarrollo, correspondiente al ejecutivo, se dio a conocer una propuesta para la aplicación de un nuevo impuesto a la enajenación e importación de combustibles fósiles de acuerdo a su contenido de carbono. Se estableció también, como mecanismo innovador con el cual es posible comprar bonos de carbono, o certificados de reducción de emisiones en el mercado de carbono, para con ello sustituir el pago del impuesto (Fanelli et al., 2015)

Sobre este último punto, los Bonos de Carbono o "Bonos Verdes", son parte de un mecanismo internacional para reducir las emisiones contaminantes del medio ambiente. Parten de la propuesta del Protocolo de Kioto cuyo objetivo es reducir el calentamiento global. De acuerdo al Chicago Climate Exchange (2017), el sistema consiste en ofrecer incentivos económicos para que las empresas privadas contribuyan a la calidad ambiental y se consiga regular la emisión generada por sus procesos productivos, considerando el derecho a emitir dióxido de carbono $\left(\mathrm{CO}_{2}\right)$ como un bien canjeable con un precio establecido.

Un bono de carbono representa el derecho a emitir una tonelada de $\mathrm{CO}_{2}$, esto permite mitigar la generación de gases de efecto invernadero, beneficiando a las empresas que no emiten o disminuyen la emisión, haciendo pagar a las que emiten más de lo permitido. Esto crea un instrumento de valor llamado Certificado de Emisiones Reducidas (CER), con el cual se deja de emitir a la atmósfera y pueden ser vendidos en el mercado de carbono a países industrializados. El funcionamiento es igual al de una bolsa de valores solo que en este caso son permisos de emisión (IMEF, 2018).

Otro factor interesante es la regulación que ejerce la Secretaría de Medio Ambiente y Recursos Naturales en México, que tiene como misión incorporar criterios e instrumentos que aseguren la óptima conservación, protección y aprovechamiento de los recursos naturales del país, conformando una política ambiental 
integral que permita alcanzar el desarrollo sustentable. Al mismo tiempo la Ley General del Equilibrio Ecológico y Protección al Ambiente (LGEEPA) garantiza el derecho constitucional de toda persona a vivir en un medio ambiente sano para su desarrollo, salud y bienestar. Algunos de sus reglamentos son en materia de impacto ambiental, de residuos peligrosos, de contaminación por ruido, de prevención y control de contaminación atmosférica, entre otras. Esta Ley fue publicada el 28 de enero de 1988 y hasta hoy se encuentra vigente. En sus estatutos no se hace referencia a tributos ambientales, sino más bien al pago de derechos por construcciones, protección de áreas verdes, etc.

Orientarse a una economía verde genera restricciones y oportunidades adicionales que no suelen ser consideradas en la literatura sobre crecimiento y cambio estructural. Las reformas fiscales ambientales pueden ayudar a poner en línea los incentivos con la meta del crecimiento verde. Este es un punto central en Latinoamérica, donde la mayoría de los países muestra relaciones altas entre el consumo de recursos naturales y el crecimiento del PIB (Banco Mundial, 2014). Según las mediciones del Banco Mundial (2017), mientras la merma o reducción del capital natural en la Unión Europea, donde se hicieron la mayoría de las reformas fiscales ambientales es de $0.2 \%$ del ingreso nacional anual, en América Latina y el Caribe esa cifra se ubica en $5.3 \%$. En contraste el nivel de emisiones per cápita en la región es de 2.92 toneladas métricas anuales de $\mathrm{CO}_{2}$, mientras que en Estados Unidos es de 7.96 toneladas. Estas diferencias indican que al fijar prioridades de política en la región recaen en la necesidad de reducir emisiones desde el punto de vista de la sostenibilidad.

\section{Conclusiones}

El objetivo de la presente investigación fue exponer las principales posturas teóricas, así como las tendencias vanguardistas que existen sobre el efecto del desempeño ambiental y el riesgo de mercado en países emergentes. A su vez, se logró contestar la pregunta: ¿cuál es el marco teórico que establece el efecto del desempeño ambiental sobre el riesgo de mercado en países emergentes?

Se puede concluir, de acuerdo a los estudios empíricos realizados a lo largo de las últimas décadas, que existe evidencia que establece que la práctica de desempeño ambiental eco-eficiente, está relacionada con la maximización de rendimientos a los accionistas a largo plazo y por lo tanto minimizando el riesgo de la acción en el mercado. Si el objetivo de la empresa es sobrevivir y prosperar, 
debe generar estrategias a largo plazo que se reflejen en la mejora ambiental y social, y esperar a cosechar los resultados.

Cabe destacar que, como primer acercamiento al tema bajo estudio, el alcance de este proyecto serán las empresas públicas que cotizan en los mercados de valores de México, Chile y Brasil en un periodo de tiempo de 10 años iniciando en el 2006. Adicional a la delimitación del alcance existen limitantes importantes al estudio. En primer lugar, los índices sustentables de los países seleccionados cuentan con diferentes metodologías, por lo que es importante analizar las principales diferencias. En segundo lugar, es importante destacar que existen periodos de incertidumbre financiera durante la limitación temporal de este proyecto. Por último, un tercer factor se relaciona con el comportamiento del mercado referente al riesgo, ya que es importante enfatizar los resultados en cuanto a industria y tamaño del mercado.

\section{Referencias}

Al-Najjar, B., \& Anfimiadou, A. (2012). Environmental policies and firm value. Business Strategy and the Environment, 21(1), 49-59.

Antolín, M. N., \& Gago, R. F. (2004). Responsabilidad social corporativa: la última innovación en management. Universia Business Review, 1(1), 28-39.

Aras, G., \& Crowther, D. (2008). Governance and sustainability: An investigation into the relationship between corporate governance and corporate sustainability. Management Decision, 46(3), 433-448.

Baron, D. P. (2007). Corporate social responsibility and social entrepreneurship. Journal of Economics \& Management Strategy, 16(3), 683-717.

Bowen, H. (1953). Social responsibility of the businessman. New York: Harper and Row.

Bragdon Jr, J. H., \& Marlin, J. A. (1972). Is Pollution Profitable?, Risk management, 19, 9-18. Carroll, A. B. (1999). Corporate social responsibility evolution of a definitional construct. Business \& society, 38(3), 268-295.

Davis, K. (1960). Can business afford to ignore social responsibilities?, California Management Review, 2(3), 70-76.

Epstein, E. M. (2007). The good company: Rhetoric or reality? Corporate social responsibility and business ethics redux. American business law journal, 44(2), 207-222.

Fanelli, J. M., Jiménez, J. P., \& López, I. (2015). La reforma fiscal ambiental en América Latina, Comisión Económica para América Latina y el Caribe (CEPAL)

Falck, O., \& Heblich, S. (2007). Corporate social responsibility: Doing well by doing good. Business Horizons, 50(3), 247-254.

Freeman, E. (1984). Strategic management: A stakeholder approach. Boston: Pitman. 
Fredrick, W. (1960). The growing concern over business responsibility. California Management Review, 2(4), 54-61.

Friedman, M. (1962). Capitalism and freedom. Chicago: University of Chicago Press.

Friedman, M. (1970). The social responsibility of business is to increase its profits. New York Times Magazine, september 13.

Henri, J. F., \& Journeault, M. (2010). Eco-control: The influence of management control systems on environmental and economic performance. Accounting, Organizations and Society, 35(1), 63-80.

Holdgate, M. W. (1996). From care to action: making a sustainable world. Taylor \& Francis.

Ingram, R. W., \& Frazier, K. B. (1980). Environmental performance and corporate disclosure. Journal of accounting research, 12(2), 614-622.

Iraldo, F., Testa, F., \& Frey, M. (2009). Is an environmental management system able to influence environmental and competitive performance? The case of the ecomanagement and audit scheme (EMAS) in the European Union. Journal of Cleaner Production, 17(16), 1444-1452.

Jaggi, B., \& Freedman, M. (1992). An examination of the impact of pollution performance on economic and market performance: pulp and paper firms. Journal of Business Finance \& Accounting, 19(5), 697-713.

Jensen, M. C., \& Meckling, W. H. (1976). Theory of the firm: Managerial behavior, agency costs and ownership structure. Journal of financial economics, 3(4), 305-360.

Jones, T. M. (1995). Instrumental stakeholder theory: A synthesis of ethics and economics. Academy of management review, 20(2), 404-437.

King, A. A., \& Shaver, J. M. (2001). Are aliens green? Assessing foreign establishments' environmental conduct in the United States. Strategic Management Journal, 22(11), 1069-1085.

Lall, S. (2001). Competitiveness indices and developing countries: an economic evaluation of the global competitiveness report. World Development, 29(9), 1501-1525.

Ley General del Equilibrio Ecológico y Protección al Ambiente

Li, W., \& Zhang, R. (2010). Corporate social responsibility, ownership structure, and political interference: Evidence from China. Journal of business ethics, 96(4), 631-645.

Mahapatra, S. (1984). Investor reaction to a corporate social accounting. Journal of Business Finance \& Accounting, 11(1), 29-40.

Margolis, J. D., \& Walsh, J. P. (2001). People and profits? The search for a link between a company's social and financial performance. Psychology Press.

McGuire, J. (1963). Business and society. New York: McGraw-Hill.

Molina, M. Charlo., \& Clemente, I. M. (2010). El comportamiento financiero de las empresas socialmente responsables. Investigaciones Europeas de Dirección y Economía de la Empresa, 16(2), 15-25.

Makower, J. (Greenbiz) (2017). The tenth annual state of green business 2017.

Marritt-Alers, K., \& Giese, G. (2013). Unlocking sustainable value in emerging markets. RobecoSam Sustainability Investing, 2012-2014. 
Murphy, C. J. (2002). The profitable correlation between environmental and financial performance: a review of the research. This document was commissioned by Light Green Advisors, Inc.

Narver, J., Slater, S., \& MacLachlan, D. (2004). Responsive and proactive market orientation and new-product success. Journal of Product Innovation Management, 21(5), 334-347.

Organización para la Cooperación y el Desarrollo Económico (2016).

Orlitzky, M., Schmidt, F. L., \& Rynes, S. L. (2003). Corporate social and financial performance: A meta-analysis. Organization studies, 24(3), 403-441.

Orsato, R. J., Garcia, A., Mendes-Da-Silva, W., Simonetti, R., \& Monzoni, M. (2015). Sustainability indexes: why join in? A study of the 'Corporate Sustainability Index (ISE)'in Brazil. Journal of Cleaner Production, 96, 161-170.

Porter, M. E., \& Van der Linde, C. (1995). Toward a new conception of the environmentcompetitiveness relationship. The journal of economic perspectives, 9(4), 97-118.

Quiroga Martínez, R. (2007). Indicadores ambientales y de desarrollo sostenible: avances y perspectivas para América Latina y el Caribe. CEPAL.

Richardson, G. (2012). Corporate social responsibility and tax aggressiveness: An empirical analysis. Journal of Accounting and Public Policy, 31(1), 86-108.

Rockness, J., Schlachter, P., \& Rockness, H. O. (1986). Hazardous waste disposal, corporate disclosure, and financial performance in the chemical industry. Advances in public interest accounting, 1(1), 167-191.

Rosewicz, B. (1990). Americans are willing to sacrifice to reduce pollution, they say. Wall Street Journal, 20, A1.

Russo, M. V., \& Fouts, P. A. (1997). A resource-based perspective on corporate environmental performance and profitability. Academy of management Journal, 40(3), 534-559.

Secretaría de Medio Ambiente y Recursos Naturales en México.

Schwab, K., \& Sala-i-Martin, X. (Eds.) The global competitiveness report 2016. Geneva: World Economic Forum.

Shane, P. B., \& Spicer, B. H. (1983). Market response to environmental information produced outside the firm. Accounting Review, 521-538.

Spicer, A. S. (2005, August). Shaping the agenda of shareholders activism: Institutional investors and global corporate social responsability. Academy of Management Proceedings (Vol. 2005, No. 1, pp. H1-H6). Academy of Management.

Standard, B., \& SERIES, A. (1977). British Standards Institution.

Stuart, H. (2011) Sustainable value presentation. Academy of management review.

Walton, C. (1967). Corporate social responsibilities. Belmont, CA: Wadsworth.

Wartick, S. L., \& Cochran, P. L. (1985). The evolution of the corporate social performance model. Academy of management review, 10(4), 758-769.

World Bank Group (Ed.). (2016). World Development Indicators 2012. World Bank Publications. 
World Bank (2014), Putting a price on carbon with a tax, World Bank.

Zhou, P., Ang, B. W., \& Poh, K. L. (2006). Comparing aggregating methods for constructing the composite environmental index: An objective measure. Ecological Economics, 59 (3), 305-311. 